\title{
REGISTRO PRELIMINAR DE MYXOMYCETOS EN LA SELVA CENTRAL DEL PERÚ
}

Reina A. Zúñiga A. ${ }^{I}$

\section{RESUMEN}

Especímenes gregarios de Arcyria denudata (L)Wettstein and Stemonitis splendens Rostafinski, fueron colectados en el valle de Chanchamayo: San Ramón y la Merced. Estas especies son descritas como contribución preliminar de Myxomycetos@̧en el Perú.

Palabras clave: Myxomycetes, Arcyria, Stemonitis, Chanchamayo.

\section{SUMMARY}

Gregarius specimens of Arcyria denudata (L) Wettstein and Stemonitis splendens Rostafinski were collected in Chanchamayo valley: San Ramón and La Merced. These especies are described as preliminary contribution of Myxomycetes in Perú.

Key words: Myxomycetes, Arcyria, Stemonitis, Chanchamayo.

Los Myxomycetos o mohos mucilaginosos, constituyen un grupo pequeño, relativamente homogéneo de organismos eucarióticos. Hasta al presente se conocen alrededor de 700 especies en este grupo. Fueron estudiados inicialmente por Link 1833, incluidos en los hongos, opinión no aceptada ampliamente por otros autores; sin embargo, hasta el presente muchos investigadores han seguido la opinión original de Link y clasifican a estos organismos incluidos en los hongos.

En nuestro país no existen estudios disponibles de este grupo de organismos, posiblemente por la poca familiaridad para reconocerlos en el estado vegetativo y/o reproductivo; deben encontrarse en las distintas regiones del país.

En la región central del país, en Chanchamayo: San Ramón y La Merced caracterizadas por su abundante vegetación, por su temperatura y humedad tropical constante, se ha colectado de la corteza de los árboles y hojas secas muchas especies de hongos y entre ellas ejemplares de Myxomycetos correspondientes a los géneros Arcyria y Stemonitis. La conservación y estudio detallado de éstos se dan a conocer en esta comunicación inicial.

\section{MATERIALES Y MÉTODOS}

El material estudiado fue colectado en una trocha a las cascadas de El Tirol, San Ramón y en un terreno de cultivo de frutales entre San Ramón y La Merced, provincia de Tarma, departamento de Junín.

En el primer caso con un cuchillo se removió parte de la corteza del árbol muerto y caído cubierto con los cuerpos fructíferos de Arcyria, visibles por su coloración rojo ladrillo intenso a rosado. En el segundo caso, los cuerpos fructíferos Stemonitis fueron colectados en condición gregaria en el haz de una hoja seca de palto, caída en el suelo húmedo, protegido por plantas de mandarina y palto.

Los especímenes fueron colectados con mucho cuidado por su condición frágil. Fueron colocados separadamente en frascos plásticos adecuados, con tapa y protegidos con papel servilleta para facilitar el transporte.

Cada recipiente conteniendo una muestra lleva una etiqueta informativa que incluye el nombre del Myxomyceto, el substrato sobre el que ha desarrollado, la localidad de colecta, fecha de colección, nombre y número del colector.

Diversos autores indican que las muestras de Myxomycetos son resistentes a los insectos y otras plagas que ata- 
can las colecciones de herbario. Para evitar estos problemas se ha colocado en cada frasco un trozo de papel filtro embebido en xilol o formol, que al evaporarse en el frasco no cerrado, permiten la muerte de los diminutos insectos.

Se ha preparado láminas temporales para la observación microscópica: reconocimiento de las distintas estructuras del cuerpo fructífero, medida e ilustración respectiva. El material estudiado se conserva en el laboratorio de botánica de la facultad de Ciencias Biológicas de la Universidad Ricardo Palma.

\section{RESULTADOS}

En las muestras colectadas se ha identificado dos géneros de Myxomycetos: Arcyria denudata (L) Wettstein y Stemonitis splendend Rostafinski. Cuya posición sistemática según Martín y Alexopoulos (1969) es la que sigue:

Orden Trichiales

Familia Trichiaceae

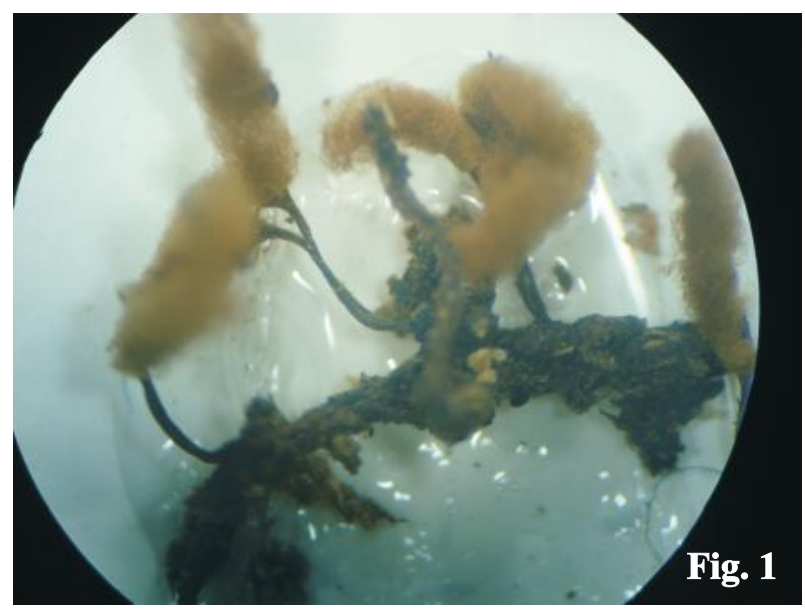

Esporangios pedicelados, gregrios

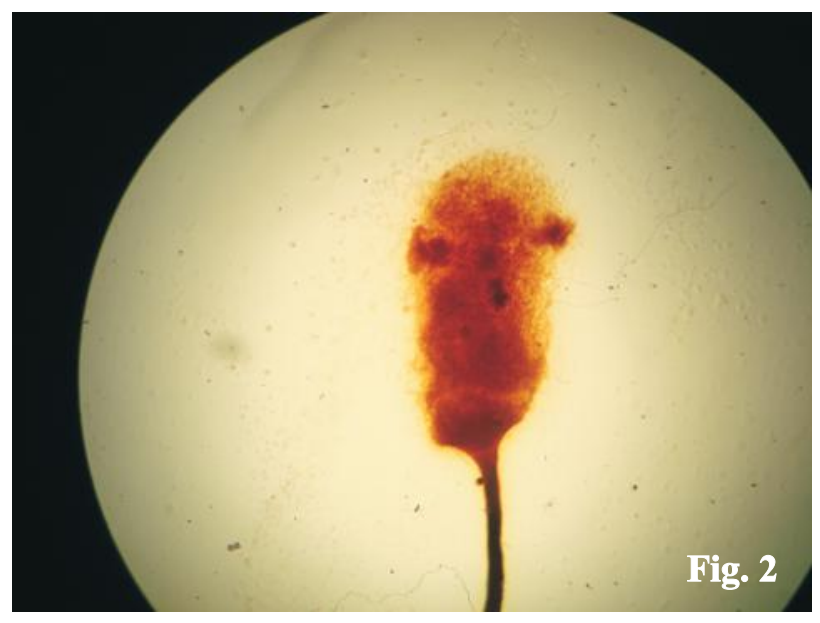

Capilicio sostenido por un calículo cónico

\section{Género Arcyria}

Especie Arcyria denudata (L) Wettstein

Especie con esporangio pedicelado (Fig. 1 y 2), gregario, generalmente cilíndrico, rosa pálido a rojo anaranjado brillante de 8001 ì a 1000ì m de diámetro y de 2.3 a 4.4 milímetros de largo, pedicelo largo cilíndrico con base cónica de 540ì m a 810 ì m de diámetro, finamente estriado, longitudinalmente, marrón oscuro de 2 a 2.30 milímetros de largo por 880ì m a 950ì m de diámetro. Peridium persistente en los cuerpos fructíferos maduros sólo a nivel del calículo cónico, marrón anaranjado intenso. Capilicio constituido por un enmarañado de filamentos de $3 i ̀ \mathrm{~m}$ diámetro con protuberancias o engrosamientos semicirculares, en conjuntos fijos a la superficie interna del calículo. Esporas ovadas, marrón

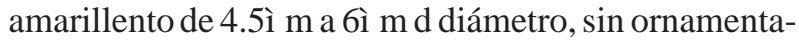
ción visible al microscopio de luz.

Substrato: habita sobre corteza húmeda de árbol muerto.

Material estudiado: R. Zúñiga 880 trocha a El Tirol, San Ramón, provincia de Tarma.

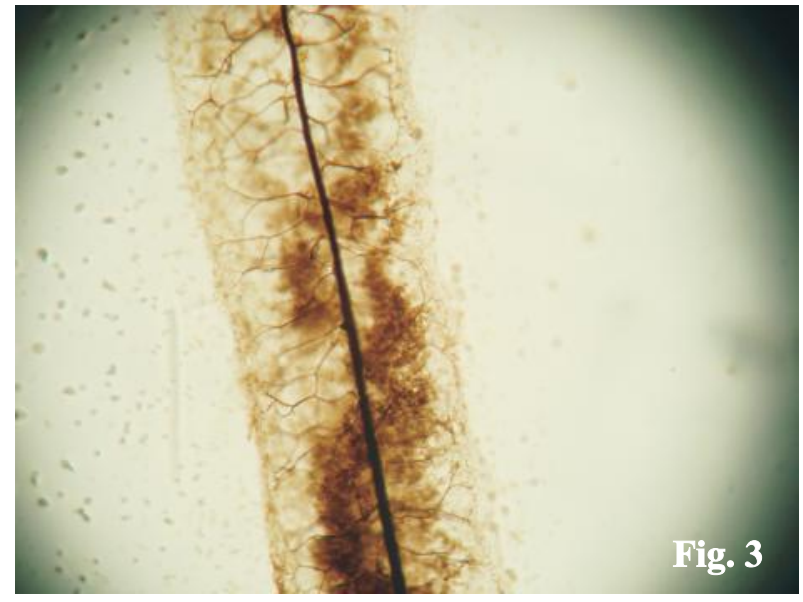

Porción de esporangio cilíndrico, con columela central

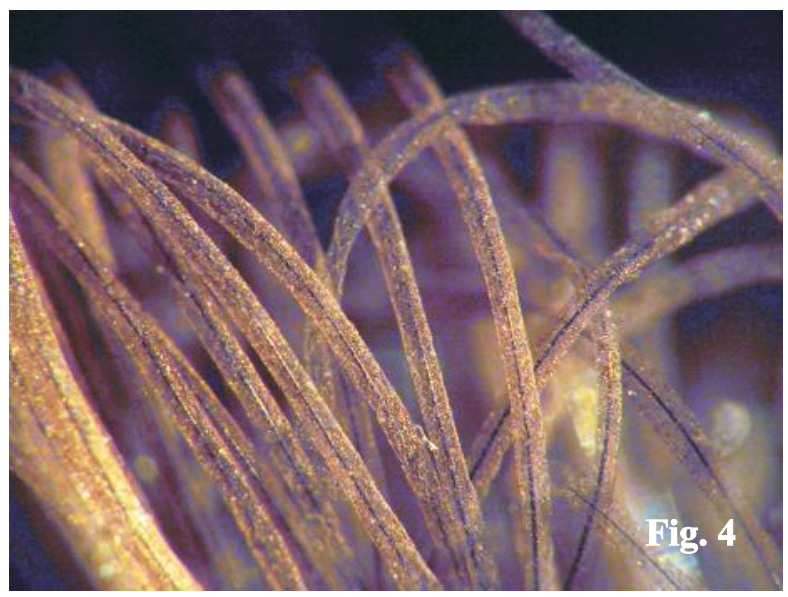

Esporangios cilíndricos, gregarios 
Orden: Stemonitales

Familia: Stemonitaceae

Género:Stemonitis

Especie: Stemonitis splendens Rostafinski

Especie con esporangios pedicelado (Fig. 3 y 4), agrupado en fascículos apretado, erguidos, rectos o curvados, cilíndricos, marrón oscuro a pálido luego de la liberación de las esporas. Pedicelo delicado negro brillante de 1.9 milímetros de largo por 610ì m de diámetro. Columela visible en el centro del esporangio, más grueso en la parte basal y progresivamente más delgado hacia el ápice, con ramas libres en toda su extensión, formando una red superficial de malla delicada. Esporas esféricas marrón pálidas de 6.9ì m a 9ì m de diámetro.

Substrato: envés de la hoja de palto, caída en suelo húmedo y protegido.

Material estudiado. R. Zúñiga 881 área de cultivo de frutales entre San Ramón y La Merced, provincia de Tarma.

\section{DISCUSIÓN}

Los Myxomycetos constituyen un grupo pequeño, relativamente homogéneo de organismos eucarióticos, representados por cerca a 700 especies desnudas, saprófitas o fagotróficas. Heinrich Link (1833) consideró a los Myxomycetes como hongos, opinión que no fue compartida por otros biólogos, Anton de Bary (1864) estudió con particular interés este grupo, lo consideró más relacionado a los protozoarios, propuso el nombre de Mycetoza, nombre usado por otros investigadores, otros siguieron la interpretación original de Link, clasificaron a los Myxomycetes como parte de los hongos. En cualquier caso, cual sea la relación con otros organismos, los MYXOMYCETES han sido, aún son estudiados por botánicos, particularmente micólogos, Martin et. al 1983, Stephenson S.L. y H. Stempen 1994.
La clasificación presente basada en el Ainsworth \& Bisby's Dictionary of the Fungus, 8th Ed. 1995. En el Dominio Eucariota, Reino Protozoa, Phylum Myxomycota o "mohos mucilaginoso plasmódicos".

\section{LITERATURA CITADA}

ALEXOPOULOS, C.J. \& MIMS, C.W., 1979, Introductory Mycology, 3rd. Ed. New York. Johns.

CARLILE, M.J., WATKINSON, S.C. \& GOODAYM, G.W. 2004, The Fungi 2nd. Ed. Elsevier Academic Press.

DE BARY, A. 1964 Die Mycetozoen (Schelimpilze), Ein Beitrag zur Kenntniss der niederten Organisen. XI + 132pp. 6pl. Engelmann, Leipzig.

HAWKSWORTH, D.L., KIRK, P.M., PEGLER, D.N. \& SUTTON, B.C. 1995 Ainsworth \& Bisby's Dictionary of the Fungi, 8th Ed. CAB International, Wallingford.

KENDRICK, B. 2000. The Fifth Kingdom Focus Publishing R. Pullino Company Newburyport MA0 1950, USA.

LINK, J.H.F. 1833 Handbuck zur Erkenung der nutzbarten und am hanfigsten workommenden Gewasche 3. Ordo fungi, Budordo 6 . Myxomycetes 405-422, 432-433 Berlin.

MARTIN, G.W. \& ALEXOPULOS, C.J., 1969. The Myxomycetes. lowa City. University of lowa Press.

ROBBRECHT, E. 1974 The genus Arcyria Wiggens (Myxomycetes) in Belguem Bulletin del Jardín Botanique Belgique 44:303-353

SMITH, H.V., SMITH A.H. \& WEBER N.S. 1981 How to know the non gilled Mushrooms. Dubuque lowa Wm, C. Brown Company Publisher.

STEPHENSON, S. \& STEMPEN, H. 1994. Myxomycetes A. Handbook of Slime Molds. Timber Press. Portland, Oregon. 